\title{
Metrology Light Source: The first electron storage ring optimized for generating coherent $\mathrm{THz}$ radiation
}

\author{
J. Feikes, M. von Hartrott, M. Ries, P. Schmid, and G. Wüstefeld* \\ Helmholtz-Zentrum Berlin (HZB), Albert-Einstein-Straße 15, 12489 Berlin, Germany \\ A. Hoehl, R. Klein, R. Müller, and G. Ulm \\ Physikalisch-Technische Bundesanstalt (PTB), Abbestraße 2-12, 10587 Berlin, Germany \\ (Received 16 July 2009; published 22 March 2011)
}

\begin{abstract}
The Metrology Light Source is a recently constructed $630 \mathrm{MeV}$ electron storage ring, operating as a synchrotron radiation source for the $\mathrm{THz}$ to extreme $\mathrm{UV}$ spectral range. It is the first storage ring optimized for generating intense, broadband, coherent $\mathrm{THz}$ radiation, based on a bunch shortening mode. Stable ("steady state") or bursting THz radiation up to an average power of about $60 \mathrm{~mW}$ can be obtained. The applied machine operation mode is achieved by manipulating the momentum compaction factor $\alpha$ by a novel tuning scheme. The underlying low- $\alpha$ scheme is of general interest for operating a storage ring in a short bunch mode and is the main subject of this paper.
\end{abstract}

DOI: 10.1103/PhysRevSTAB.14.030705

PACS numbers: 29.20.db, 41.60.Ap

\section{INTRODUCTION}

There is an increasing interest in generating picosecond and subpicosecond electron bunches in storage rings, for an overview see [1-3], stimulated by two quite different applications. Short electron bunches produce short $\mathrm{x}$-ray pulses, very suitable for time resolved measurements. These are becoming of increasing interest in many fields of research. The second interest in short bunches is the intense, coherent broadband $\mathrm{THz}$ radiation $[4,5]$ emitted by these bunches [6-9]. In this frequency range, sometimes called the "THz gap," there is a shortage of intense, broadband sources.

The Metrology Light Source (MLS) [10,11] of the PTB is dedicated to metrology and scientific developments in the THz to extreme UV spectral range. It was designed, built, and is operated in close cooperation with the HZB, formerly BESSY. To cover the tasks in the THz range it is optimized to shorten bunches into the ps range using a flexible low- $\alpha$ optics operation mode. The emitted $\mathrm{THz}$ radiation is made up of single-cycle pulses with a $500 \mathrm{MHz}$ repetition rate and a high average power level. Bursting and stable "steady state" THz radiation is generated. An extremely stable steady state mode is expected, excellently suited for spectroscopic applications, as achieved at BESSY II $[12,13]$.

The key parameter for shortening the bunch length is the momentum compaction factor $\alpha$, a tunable, momentum

\footnotetext{
*Corresponding author. godehard.wuestefeld@helmholtz-berlin.de
}

Published by American Physical Society under the terms of the Creative Commons Attribution 3.0 License. Further distribution of this work must maintain attribution to the author(s) and the published article's title, journal citation, and DOI. dependent storage ring parameter which strongly affects the longitudinal bunch focusing. The "zero current" bunch length scales within limits with $\sqrt{\alpha}$. A precise control of the momentum dependence of $\alpha$ is required, to store short bunches in a low- $\alpha$ optics with good beam lifetime.

Electron storage rings optimized for $\mathrm{THz}$ generation are suggested in [14-18]; for low- $\alpha$ operation see $[19,20]$. Recently, low- $\alpha$ optics were investigated at several light sources [1], where the longitudinal chromatic tuning of the

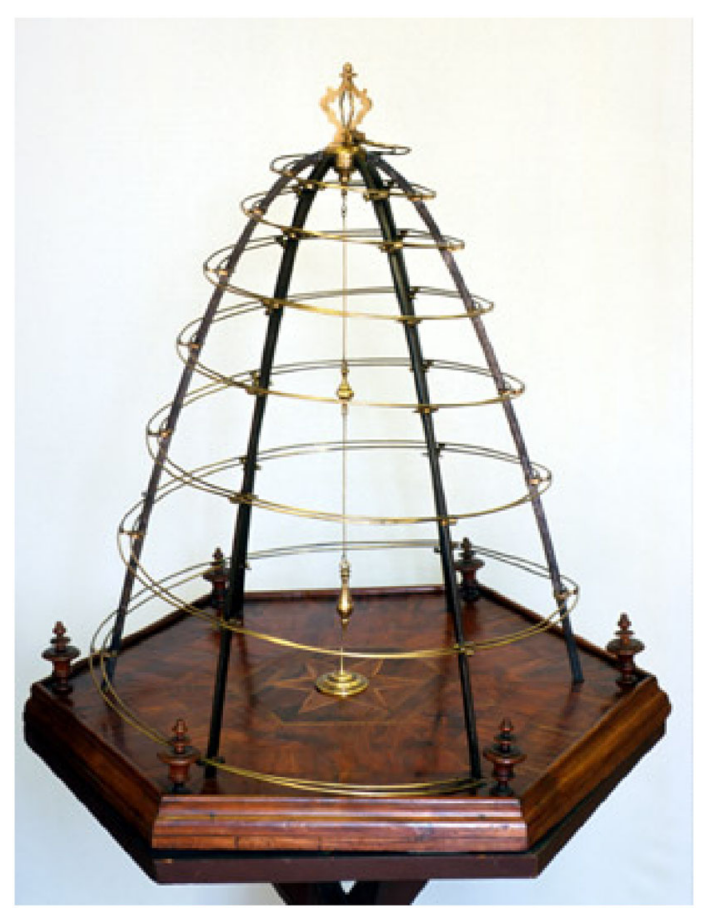

FIG. 1. Isochronous apparatus proposed 1699 by Jean Truchet [23]. 
low- $\alpha$ optics to some extent is supported by the existing harmonic sextupoles. The MLS is the first storage ring, which applies a new and dedicated method for reliable and easy tuning of the low- $\alpha$ optics. For the first time, a combination of sextupoles and octupoles is applied to correct higher orders of $\alpha$ [15]. With this method the electron beam can be stored at positive or negative $\alpha$ values.

The main topic of this paper is the low- $\alpha$ optics and its properties, discussed in the Sec. II, where the main MLS parameters are presented. This section has two subsections, the first describes results of tuning the low- $\alpha$ optics and the second describes longitudinal beam dynamics. The special aspect of the beam dynamics is the combined effect of sextupoles and octupole on the buckets; they control the momentum dependent higher orders of $\alpha$ (see below). With these magnets the tuning and operation of the low- $\alpha$ optics becomes very reliable. Section III presents some first results on coherent radiation, i.e., the production of stable and bursting $\mathrm{THz}$ radiation, to demonstrate that the main goals of $\mathrm{THz}$ production are achieved.

Historical detour.-A beam optics tuned into a low- $\alpha$ mode is also called an "isochronous optics." All particles, independent of their energy, oscillate with the same revolution time around the machine. The first proposal to exactly tune an optics in this mode, covering the range from nonrelativistic to relativistic particle motion, was done by Thomas [21]. With his results the range of the isochronous cyclotron to higher beam energies could be extended. His paper became classified in the U.S. during the times of World War II [22].

Further back in history, the first mechanical model of an isochronous apparatus was suggested 300 years ago by Jean Truchet [23], see Fig. 1, to demonstrate Galileo's law of falling bodies along an inclined plane. The gain of velocity of a downhill running ball is exactly balanced by increasing the orbit length to preserve a constant revolution time. This is an example for nonrelativistic particles.

In the high energy limit the conditions are different. For highly relativistic particles velocity dependent effects can be ignored. Their revolution time around a storage ring is only path length dependent. For an isochronous storage ring of constant revolution time, as discussed here, the orbit lengths have to be independent of energy. This is achieved by the low- $\alpha$ optics.

\section{THE MLS MACHINE}

The MLS lattice consists of four double bend achromatic cells (DBA), see Fig. 2. Details of the beam optics are shown in Fig. 3; the main parameters are listed in Table I. We call "user optics" a machine setup without any special attention to the longitudinal bunch length. This is the standard operation mode of the MLS. The "low- $\alpha$ " optics is a machine setup, where we are especially interested in the length of the bunches. This is not a unique setup;

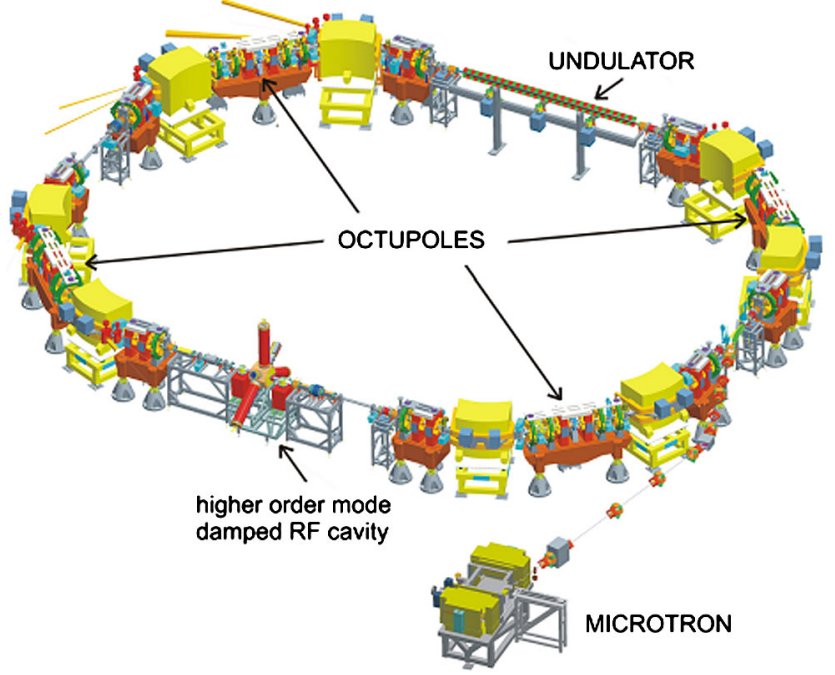

FIG. 2. Scheme of the MLS machine.
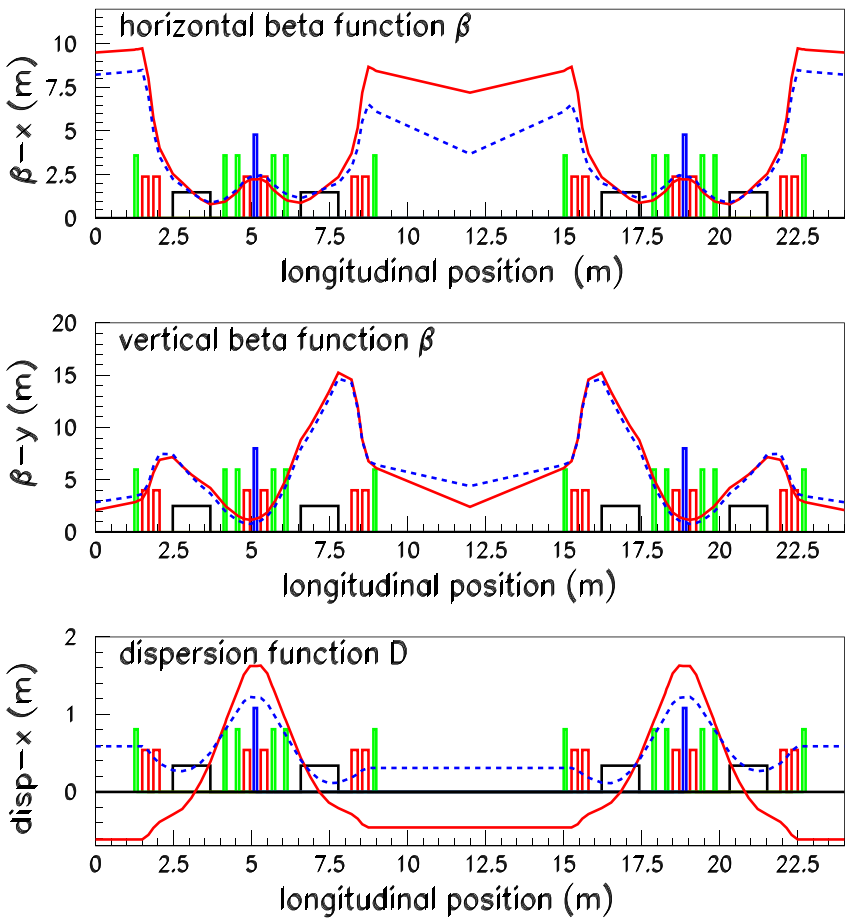

FIG. 3. Optical functions of the user (blue, dotted line) and an $\alpha=0$ optics (red line). Half of the ring is displayed. Magnetic multipoles are indicated by colored boxes with increasing height, dipoles (black), quadrupoles (red), sextupoles (green), and octupoles (blue).

several parameters can be varied, like the beam energy, the $\alpha$ value, or the rf voltage. Depending on the machine tuning, we expect zero current bunch lengths (rms) of $22 \mathrm{ps}$ for the user optics to less than 1 ps in the low- $\alpha$ optics. The achievable lower value is still unknown; it depends on several limiting effects [1]. The MLS is equipped with a novel, higher order mode damped rf cavity [24]. 
TABLE I. Main MLS user optics parameters.

\begin{tabular}{lc}
\hline \hline Parameter & Value \\
\hline Injection energy & $105 \mathrm{MeV}$ \\
Maximum energy $E_{0}$ & $630 \mathrm{MeV}$ \\
Circumference $L_{0}$ & $48 \mathrm{~m}$ \\
Revolution frequency $f_{\text {rev }}$ & $6.25 \mathrm{MHz}$ \\
rf frequency $f_{\text {rf }}$ & $500 \mathrm{MHz}$ \\
rf voltage $V_{\text {rf }}$ & $325 \mathrm{kV}$ \\
Horizontal/vertical tunes & $3.18 / 2.23$ \\
Short/long straight & $2.5 \mathrm{~m} / 6 \mathrm{~m}$ \\
Natural emittance & $110 \mathrm{~nm} \mathrm{rad} \mathrm{@630} \mathrm{MeV}$ \\
Relative, natural energy spread & $4.4 \times 10^{-4} @ 630 \mathrm{MeV}$ \\
Longitudinal damping time & $11 \mathrm{~ms} @ 630 \mathrm{MeV}$ \\
Transverse damping time & $22 \mathrm{~ms} @ 630 \mathrm{MeV}$ \\
Momentum compaction factor $\alpha$ & 0.033 \\
Energy loss/turn & $8 \mathrm{keV} \mathrm{@630} \mathrm{MeV}$ \\
Dipole bending radius & $1.53 \mathrm{~m}$ \\
Dipole vacuum chamber full height & $42 \mathrm{~mm}$ \\
\hline \hline
\end{tabular}

The rf system has a large voltage tuning range up to $500 \mathrm{kV}$ for additional easy bunch length manipulation. (Bunch length manipulations at the former BESSY I for longer bunches of 20 to 240 ps by different rf parameters are discussed in [25].)

To operate the low- $\alpha$ mode, the beam is injected from a $105 \mathrm{MeV}$ microtron into the storage ring and ramped to the specified energy by applying the user optics. Then a sequence of different magnet optics settings is applied, to stepwise reduce $\alpha$ to the target value. An automated ramp procedure controls all these steps, from the injection to the desired energy and $\alpha$ value, including the required orbit correction. This simplifies the low- $\alpha$ operation. To avoid lifetime reductions or beam loss, these files are set up for a fixed transverse working point and small chromaticities.

\section{A. The low- $\alpha$ optics}

The momentum compaction factor $\alpha$ is defined by the relative change of the particle path length around the storage ring $\left(L-L_{0}\right) / L_{0}$ as a function of momentum deviation $\delta=\left(p-p_{0}\right) / p_{0}$ [26]

$$
\left(L-L_{0}\right) / L_{0}=\alpha \delta .
$$

To avoid additional amplitude dependent orbit lengthening effects, the transverse chromaticity has to be set to small values [27-29]. Alpha is considered as a function of $\delta$ only and expanded into a power series, using the notation [30,31]

$$
\alpha=\alpha_{0}+\alpha_{1} \delta+\alpha_{2} \delta^{2} \ldots
$$

A properly designed low- $\alpha$ optics requires the control of the momentum dependent synchrotron oscillation frequency $f_{s}$ if critical resonances, such as $f_{s}=0 \mathrm{kHz}$ (respectively $\alpha=0$ ), are approached. The control of $f_{s}$ is achieved by tuning the three leading $\alpha$ terms, by applying the relation $f_{s} \propto \sqrt{\alpha}$ at different rf frequencies. An approximate hierarchy exists for correcting these terms; see comment in [31]. The leading term $\alpha_{0}$ is controlled by the linear dispersion function $D$ inside the dipoles of bending radius $\rho, \alpha_{0}=\oint(D / \rho) d s / L_{0}$, where $s$ is the longitudinal orbit position. The next term, $\alpha_{1}$, is proportional to the longitudinal chromaticity, $\xi_{s}=\left(\partial f_{s} / \partial \delta\right) / f_{\text {rev }}$ at $\delta=0$, and controlled at fixed transverse chromaticities by three strategically placed sextupole families [32-34]. Furthermore, the curvature of $\alpha$, given by $\alpha_{2}$, is controlled by suitably placed octupoles.

For the design of the MLS ring, computer simulations with the optics code MAD [35] were performed to optimize the location and strength of these correction elements (concerning $\alpha_{2}$ simulations see [31]). If only two transverse chromatic sextupole families are applied for a low- $\alpha$ optics, $\alpha$ shows a strong dependence on momentum, case A of Fig. 4. Already at a small momentum deviation of $\delta=0.001$ the value $\alpha=0$ is reached, which limits the stable dynamic aperture. This is typical for existing storage rings, where only the transverse chromaticity correction is foreseen and the longitudinal chromaticity stays uncorrected. With an additional 3rd sextupole family all three chromaticities can be corrected independently, allowing one to set up a low- $\alpha$ optics. The momentum acceptance of the lattice becomes drastically enlarged for $\xi_{s}=0$, case B. The curve B does not hit the $\alpha=0$ axis within the shown momentum range. However, because of its weak negative curvature, the tails of line B will hit the $\alpha=0$ axis within the shown range, if $\alpha_{0}$ is further approaching zero. The momentum values at these crossing points define the momentum acceptance. Reducing $\alpha_{0}$ further lowers the acceptance. To overcome this limitation, a single octupole family is added to control the curvature of $\alpha$ with respect to the momentum, to keep particles in the momentum halo of the beam away from the $\alpha=0$ resonance, case C.

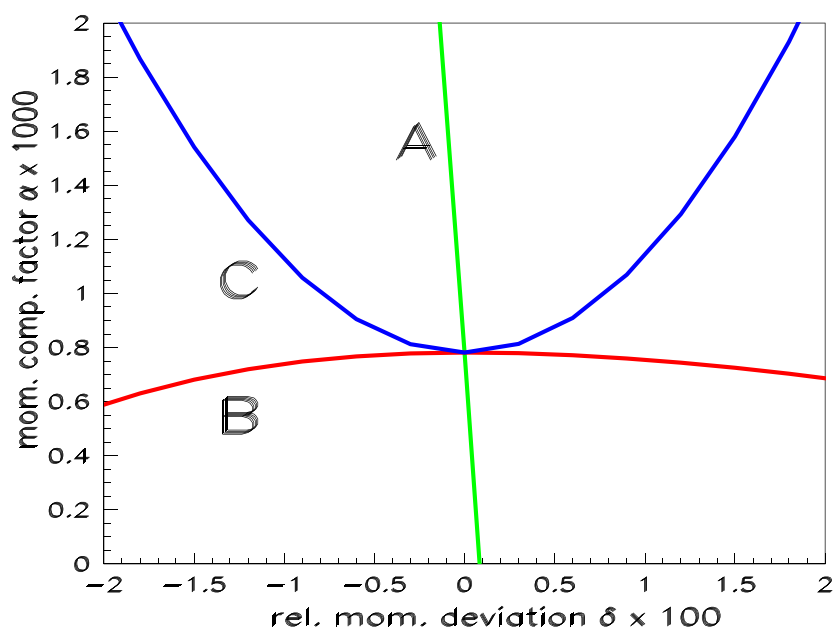

FIG. 4. Simulated, nonlinear $\alpha$ of the MLS at $\alpha_{0}=8 \times 10^{-4}$ as a function of momentum and different correction magnets. The labels are A two transverse chromatic sextupole families, B three chromatic sextupoles families, and $\mathrm{C}$ an additional octupole family. 
For $\alpha_{0}>0$ a positive curvature is required and vice versa when $\alpha_{0}$ is negative (the situation becomes more complex in case of $\alpha$ buckets, see below). Presently, the $\alpha_{0}>0$ mode is mostly applied.

It was found by MAD simulations that one octupole family is sufficient to preserve a good transverse dynamic aperture when placed in the center of the achromat, at maximum dispersion, see Fig. 3. The octupoles are $100 \mathrm{~mm}$ long, have an aperture radius of $43 \mathrm{~mm}$ and a maximum pole tip field of $0.03 \mathrm{~T}$, at a nominal excitation current of $6 \mathrm{~A}$. Figure 5 shows measurements of $f_{s}$ as a function of the $\mathrm{rf}$ frequency change, $\Delta f_{\mathrm{rf}}$, at $630 \mathrm{MeV}$ beam energy, $250 \mathrm{kV}$ rf voltage, and $\alpha_{0}=0.00046$, a typical parameter set to produce $3 \mathrm{ps}$ (rms) long bunches. This figure corresponds to the $\alpha=\alpha(\delta)$ presentation of Fig. 4. As was predicted by simulations, the MLS optics can be tuned to $\xi_{s}=0$ by the sextupoles and the octupoles control the curvature of $f_{s}$ with respect to $\Delta f_{\text {rf }}$. The octupole excitation current was $-6 \mathrm{~A}$ in this example. This figure also shows that the residual octupole content of the machine lattice is small. There is nearly no curvature if the octupole fields are switched off. We did not try to estimate residual octupole effects during the design phase of the MLS, but relied on sufficiently tunable octupoles to control the $\alpha$ curvature. A negative curvature can be achieved by powering the octupoles in the opposite way and is required for negative low- $\alpha$ operation.

Lifetime effects of the octupoles in different low- $\alpha$ settings are shown in Fig. 6. For a multibunch beam of about $15 \mathrm{~mA}$ at $630 \mathrm{MeV}$ beam energy and $250 \mathrm{kV}$ rf voltage, the lifetime was measured for different excitation currents of the octupoles, ranging from $-6 \mathrm{~A}$ to $+6 \mathrm{~A}$.

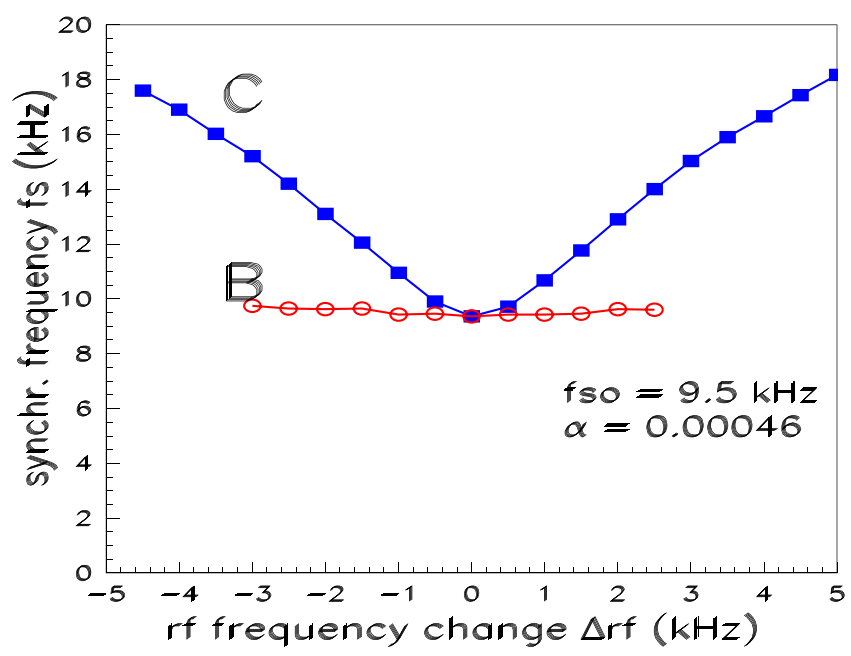

FIG. 5. Measured synchrotron frequency $f_{s}$ as a function of the rf frequency change $\Delta f_{\text {rf }}$ for $\alpha_{0}=4.6 \times 10^{-4}$ and $f_{s}=$ $9.5 \mathrm{kHz}$. Data points are connected by lines to guide the eye. The labels are B three chromatic sextupoles families "on" and octupoles "off," C three sextupole and one octupole family "on." A value of $\alpha_{2}=3$ for case $\mathrm{C}$ is estimated by a fit.

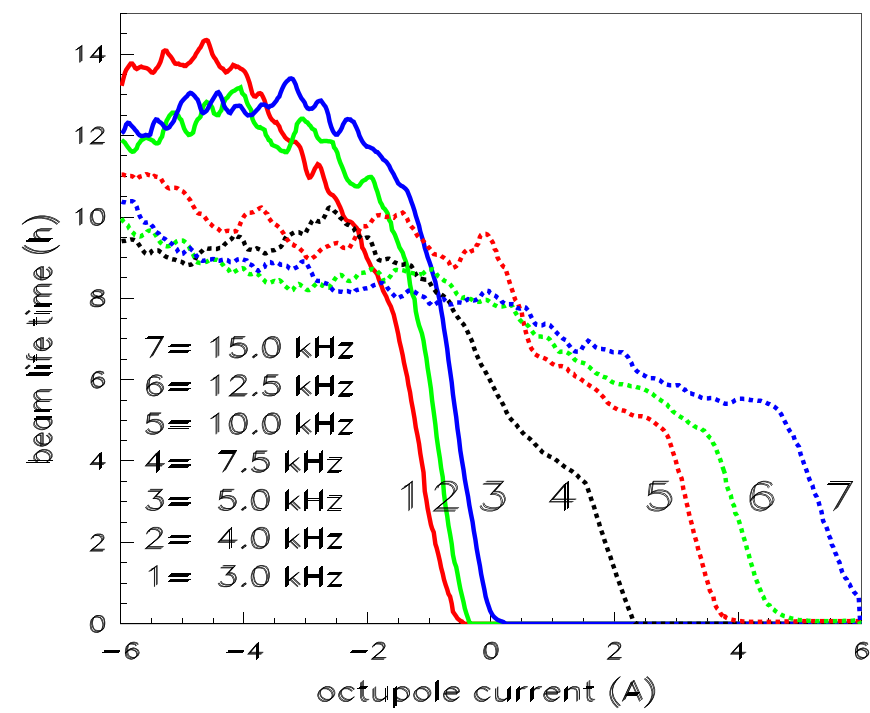

FIG. 6. Beam lifetime as a function of octupole currents and different low- $\alpha$ values. The low- $\alpha$ values are indicated by the synchrotron frequency.

The low- $\alpha$ optics is characterized by the synchrotron frequency shown in the figure, for example, $15 \mathrm{kHz}$ is related to $\alpha_{0}=1 \times 10^{-3}$ and $3 \mathrm{kHz}$ to $\alpha_{0}=4 \times 10^{-5}$. Without or with octupole fields powered with positive currents, values of $f_{s}<7.5 \mathrm{kHz}$ cannot be reached. This case is similar to curve $\mathrm{C}$ of Fig. 4, but with opposite, negative curvature of $\alpha(\delta)$, bended the other way around with respect to the $\alpha=0$ axis. In turn, $\alpha$ as a function of momentum shows then two zero crossings, defining two unstable fixed points limiting the momentum acceptance. The appearing bucket is of type $\mathrm{C}$ as shown in Fig. 8, because $\alpha_{0}$ and $\alpha_{2}$ are of opposite sign. With positively curved $\alpha(\delta)$, where $\alpha_{0}$ and $\alpha_{2}$ are positive, presented as curve $\mathrm{C}$ in Fig. 4, even the lifetime improves at values $f_{s}<7.5 \mathrm{kHz}$. We expected this improvement because beam particles in the momentum halo are kept away from $\alpha=0$. This scheme is described by bucket type A of Fig. 8 for positive values of $\alpha_{0}$ and $\alpha_{2}$. The small amplitude oscillations are not affected in the presented tuning range, i.e., the stable fixed point stays at the same phase point because $\alpha_{0}$ was kept positive. These results are in agreement with the MLS design report [15]. To achieve these very low $\alpha$ values it would not be sufficient to tune the higher orders of $\alpha$ to zero, as discussed in [36]. A tuning to smaller low- $\alpha$ values at still good lifetimes is possible by increasing further the quadrupole "q1" (placed inside the achromat) current, see Fig. 7, but the synchrotron frequency was no longer visible. (Recently, octupoles are also foreseen for the NewSUBARU ring, partly to improve the low- $\alpha$ operation [37].)

The momentum compaction factor as a function of the quadrupole "q1" family excitation current at $630 \mathrm{MeV}$ is shown in Fig. 7, where $\alpha_{0}$ is derived by scaling the measured $f_{s}$ value, $\alpha_{0} \propto f_{s}^{2}$. Alpha can be varied by more than 


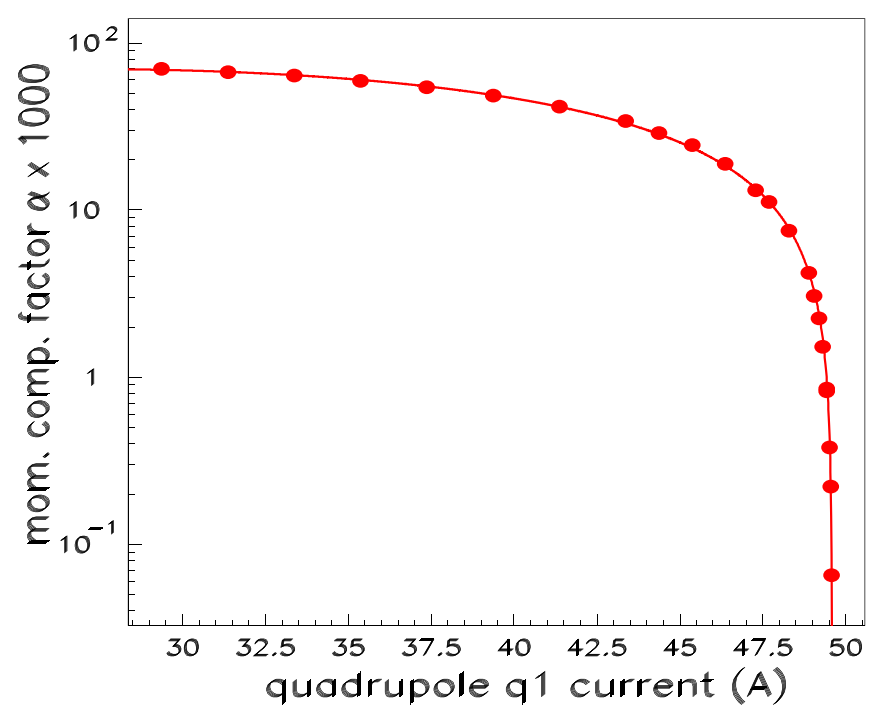

FIG. 7. Measured low- $\alpha$ tuning range $\left(\alpha_{0}>0\right)$ as a function of the quadrupole "q1" current with a parabolic fit.

a factor of 1000. Presently, currents of about $150 \mathrm{~mA}$ with few hours lifetime can be stored in low- $\alpha$ optics at 630 , 450,350 , or $250 \mathrm{MeV}$. For the first time, low- $\alpha$ optics and $\mathrm{THz}$ generation are explored at such low energies, which offers the chance to obtain information of the minimum machine energy required for a reliable $\mathrm{THz}$ operation.

To focus bunches to 3 ps length, the MLS and the $1.7 \mathrm{GeV}$ BESSY II [38] rings have both to be tuned into a low- $\alpha$ optics. Because of the different machine parameters of the MLS, the required $\alpha$ could be 1 order of magnitude larger, $\alpha=6 \times 10^{-4}$ instead of $\alpha=4 \times 10^{-5}$ at BESSY II. The larger $\alpha$ value of the MLS leads to advantages in orbit stability compared to the BESSY II ring [39].

\section{B. Longitudinal bucket dynamics}

Some basic properties of the longitudinal beam dynamics [26] can be discussed with the two dominant coefficients of $\alpha, \alpha=\alpha_{0}+\alpha_{2} \delta^{2}$. The $\alpha_{1}$ term is suppressed at zero longitudinal chromaticity by sextupoles. Beam dynamics with non-negligible $\alpha_{1}$ are discussed in [30-34,40], and recently [41] for $\alpha_{1}=0$. The Hamiltonian $H$ of the single particle coordinate variables $(\phi, \delta)$, where $\phi$ is the longitudinal phase position of the particle with respect to the rf phase, can be approximated for nonaccelerated, relativistic particles as

$$
H=H_{0} \cos (\phi)+2 \pi f_{\mathrm{rf}}\left(\alpha_{0} \delta^{2} / 2+\alpha_{2} \delta^{4} / 4\right),
$$

with $H_{0}=e V_{\text {rf }} f_{\text {rev }} / E_{0}$ and $E_{0}$ the total particle energy. A Hamiltonian of this type describes a form of Duffing oscillator [42]. Properties of this Hamiltonian are characterized by stable and unstable fixed points (FP), $\operatorname{FP}\left(\phi_{F}, \delta_{F}\right)$. The phase related FP are $\phi_{F}=0$ and $\phi_{F}=$ $\pm \pi$. They are located at the node of the rf voltage amplitude, because there is no acceleration. The momentum
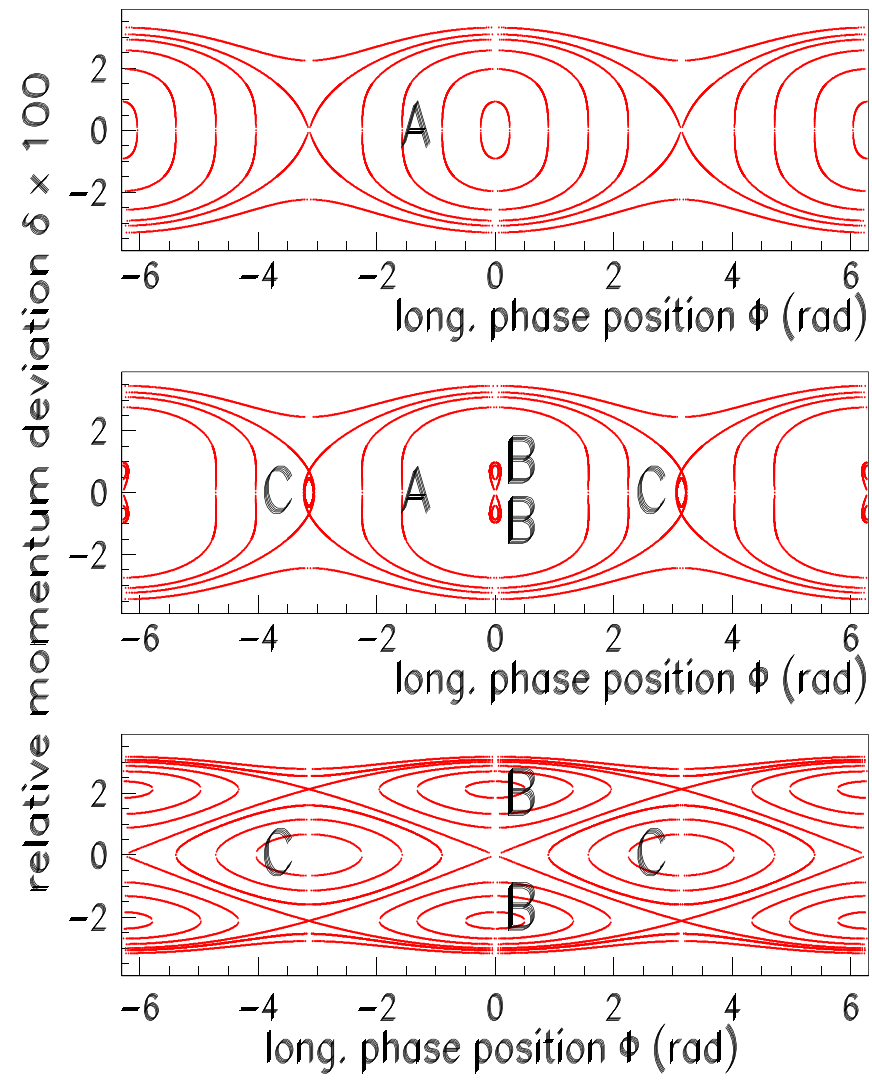

FIG. 8. Longitudinal phase space figures of the bucket shape evolution at the transition from $\alpha_{0}>0$ (upper part of the figure) to $\alpha_{0}<0$ (middle and lower part of the figure). The shapes are generated by varying $\alpha_{0}$ and $\alpha_{2}$.

related FP-part $\delta_{F}$ is derived by solving $\partial H / \partial \delta=0$, yielding two solutions, $\delta_{F}=0$ and $\delta_{F}= \pm \sqrt{-\alpha_{0} / \alpha_{2}}$. The second solution is real, if $\alpha_{0}$ and $\alpha_{2}$ are of opposite signs. Within the interval $-\sqrt{-\alpha_{0} / \alpha_{2}}<\delta<\sqrt{-\alpha_{0} / \alpha_{2}}$, the sign of $\alpha(\delta)$ agrees with the sign of $\alpha_{0}$. Particles perform stable oscillations around the stable FP. The maximum of the oscillation amplitude is limited by the unstable FP.

Qualitative bucket dynamics.-New properties of the bucket arise, if $\alpha_{0}$ is tuned from positive to negative values, at $\alpha_{2}>0$. Typical examples of the bucket appearance during this $\alpha_{0}$ zero crossing are shown in Fig. 8, based on numerical simulations. For $\alpha_{0}>0$ there is a bucket around the stable $\operatorname{FP}(0,0)$, indicated as bucket A, Fig. 8 (top). Some properties of this bucket, like synchrotron oscillation frequency and small amplitude bunch length, are closely related to the case $\alpha_{2}=0$ and only $\alpha_{0}$ is nonvanishing. If $\alpha_{0}$ passes zero to negative values, the stable FP of bucket A becomes unstable and a pair of new buckets, named $\mathrm{B}$, around the stable $\operatorname{FP}\left(0, \pm \sqrt{-\alpha_{0} / \alpha_{2}}\right)$ are created, see Fig. 8 (middle). The two buckets B are of " 8 " shape. Simultaneously, the bucket $\mathrm{C}$ appears, centered around $\operatorname{FP}( \pm \pi, 0)$. In the beginning of the transition the core of bucket $\mathrm{A}$ is affected by 
this change. But finally, at further reduced $\alpha_{0}$ values, there is a special parameter set where bucket $\mathrm{A}$ is completely filled with the buckets B, Fig. 8 (bottom). During the zero crossing of $\alpha_{0}$ the beam moves from bucket $\mathrm{A}$ to $\mathrm{B}$, smoothly changing its $\delta_{F}$ value but at fixed $\phi_{F}$ value.

The indication of this transition is the appearance or disappearance of the double beam. This is visible on a synchrotron radiation monitor [43], Fig. 9, which shows a transverse cross section of the beam. At negative $\alpha_{0}$ and $\alpha_{2}>0$ the stored electron beam splits into two stable bunches ( $\alpha$-bucket storage) of good beam lifetime, even at $150 \mathrm{~mA}$ beam current. The value of $\alpha_{0}$ can be shifted back and forth around zero to toggle the beam between the double and single bucket. Based on MAD simulations, we expect that small changes in $\alpha_{0}$ do not effect slope and curvature of $\alpha$ with respect to the momentum. The $\alpha$ buckets were first measured by [40]. In this quoted paper the discussed double beam storage is based on a slightly different process, based on the appearance of $\alpha_{0}$ and $\alpha_{1}$ buckets.

The energy of the two bunches of the double beam can be absolutely measured with a Compton-backscattering technique [10], by using a $\mathrm{CO}_{2}$ laser, Fig. 10. From the cutoff edges in the spectrum of the backscattered photons the bunch energy can be determined.

The spectrum analyzer signal of $f_{s}$ vanishes if $\alpha_{0}$ approaches zero. With the appearance of the double buckets the $f_{s}$ signal, now one for each bucket, becomes visible again. During this transition the emitted coherent $\mathrm{THz}$ power was detected. It reaches first a maximum intensity and then decreases due to the two $\alpha$-bucket populations. The appearance of the stable double buckets simplifies the tuning of low- $\alpha$ optics to smallest $\left|\alpha_{0}\right|$ value and demonstrates the flexibility of the low- $\alpha$ optics, achieved by sextupole and octupole magnets.

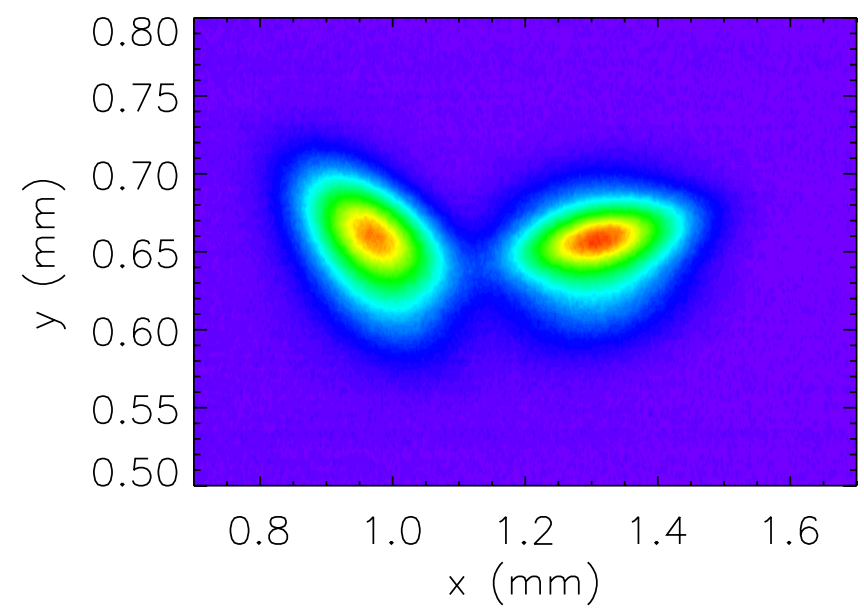

FIG. 9. Stable beam storage in two buckets for $\alpha_{0}<0$ and $\alpha_{2}>0$. Visible is the transverse beam cross section in a dispersive location on a synchrotron radiation monitor. The bunches are tilted by spurious beam optics coupling effects.

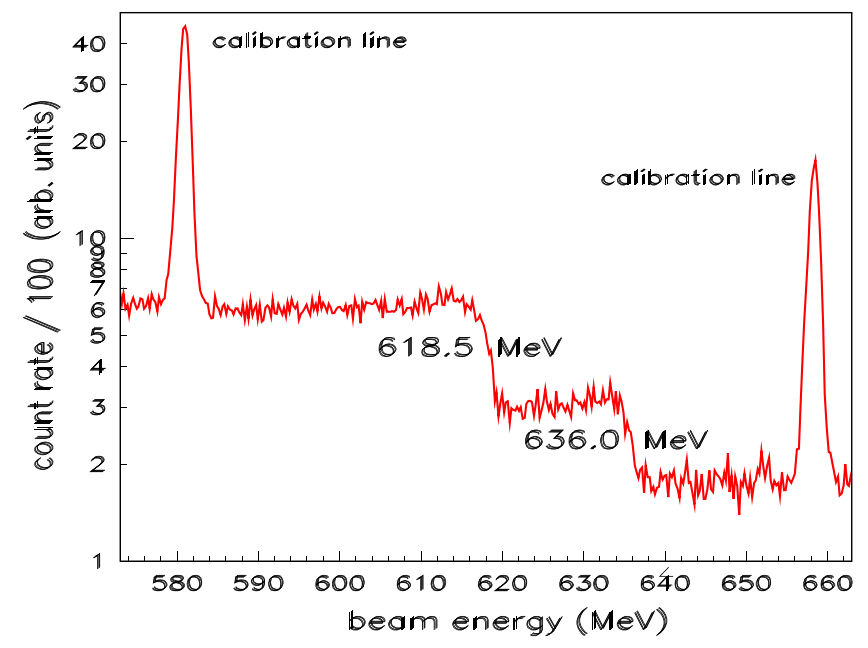

FIG. 10. Absolute energy measurement of the double beam by Compton backscattering. The relative energy uncertainty is about $2 \times 10^{-4}$. The measured spectrum shows the number of backscattered photons as a function of the photon energy. The photon energy is here already expressed as electron beam energy values. The two cutoff edges are related to the two beam energies, as indicated in the figure.

Small amplitude bucket dynamics.-In a quantitative analysis some properties of these buckets are derived. To calculate synchrotron oscillation frequencies and relations between bunch length and momentum, a small amplitude approximation of the Hamiltonian is applied. The Hamiltonian around the FP is expanded and only second order terms in $\bar{\phi}=\phi-\phi_{F}$ and $\bar{\delta}=\delta-\delta_{F}$ are kept. The resulting Hamiltonian $H_{\text {app }}$ is given as

$$
H_{\text {app }}=H_{0} \bar{\phi}^{2} / 2+2 \pi f_{\text {rf }} \bar{\delta}^{2}\left(\alpha_{0}+3 \alpha_{2} \delta_{F}^{2}\right) / 2 .
$$

This Hamiltonian describes an oscillator of synchrotron frequency $f_{s}$, with

$$
f_{s}^{2}=f_{\mathrm{rf}} H_{0}\left(\alpha_{0}+3 \alpha_{2} \delta_{F}^{2}\right) /(2 \pi) .
$$

For stable oscillation $f_{s}$ has to be real, demanding a correct sign relation between $\alpha_{0}, H_{0}$ and the chosen FP phase $\phi_{F}=0$ or $\pm \pi$ of the expansion. A summary of the small amplitude synchrotron oscillation for the three bucket types is given in Table II.

For the small amplitude expansion a relation between $\bar{\phi}$ and $\bar{\delta}$ can be derived by equalizing the potential $(\bar{\delta}=0)$ and kinetic $(\bar{\phi}=0)$ energy part of the Hamiltonian $H_{\text {app }}$, yielding

$$
\bar{\phi}^{2}=2 \pi f_{\mathrm{rf}} \bar{\delta}^{2}\left(\alpha_{0}+3 \alpha_{2} \delta_{F}^{2}\right) / H_{0} .
$$

For the different bucket types and related FP, the ratio $(\bar{\phi} / \bar{\delta})^{2}$ is given in Table II. The small amplitude values $(\bar{\phi}, \bar{\delta})$ can also be interpreted as zero current values of the rms bunch length $\bar{\phi}$ and rms momentum spread $\bar{\delta}$. As a result of this analysis, it is shown that the bunch length inside the " 8 "-shaped buckets B becomes $\sqrt{2}$ times longer 
TABLE II. Small amplitude relation between oscillation amplitude $\bar{\phi}$, momentum deviation $\bar{\delta}$, and the synchrotron frequency $f_{s}$ for $\alpha_{2}>0$. These relations are calculated at the given, stable fixed points.

\begin{tabular}{lcccc}
\hline \hline Bucket & $\operatorname{FP}\left(\phi_{F}, \delta_{F}\right)$ & $\alpha_{0}$ & $(\bar{\phi} / \bar{\delta})^{2}$ & $f_{s}^{2}$ \\
\hline A & $(0,0)$ & $>0$ & $2 \pi f_{\text {rf }} \alpha_{0} / H_{0}$ & $f_{\text {rf }} H_{0} \alpha_{0} /(2 \pi)$ \\
B & $\left(0, \sqrt{-\alpha_{0} / \alpha_{2}}\right)$ & $<0$ & $4 \pi f_{\text {rf }} \alpha_{0} / H_{0}$ & $2 f_{\mathrm{rf}} H_{0} \alpha_{0} /(2 \pi)$ \\
C & $( \pm \pi, 0)$ & $<0$ & $2 \pi f_{\text {rf }} \alpha_{0} / H_{0}$ & $f_{\text {rf }} H_{0} \alpha_{0} /(2 \pi)$ \\
\hline \hline
\end{tabular}

for the same momentum spread, compared to buckets A and $\mathrm{C}$, which are the better choice for short bunches. Different to the maximum oscillation amplitude (see below), the relation between momentum spread and bunch length depends for all bucket types on $\alpha_{0}$ and the $\mathrm{rf}$ voltage, introduced by $H_{0}$.

Bucket size limits.-A further analysis is done to derive relations for maximum bunch length $\phi_{\max }$ and momentum acceptance $\delta_{\max }$ from the unexpanded Hamiltonian $H(\phi, \delta)$ of Eq. (3). These maxima are located on the separatrix and are half width values. For example, the value of $H$ on the separatrix of bucket $\mathrm{B}, H_{\mathrm{B}}$, is calculated at the unstable $\operatorname{FP}(0,0)$, yielding $H_{B}=$ $H(0,0)=H_{0}$. In the present context, the rf voltage is defined in such a way that $H_{0}<0$. The $\phi_{\max }$ term is derived from $H\left(\phi_{\max }, \delta_{F}\right)=H_{\mathrm{B}}$, where $\delta_{F}^{2}=-\alpha_{0} / \alpha_{2}$ is the value of the stable FP, a location of zero kinetic oscillation energy. The solution is $\cos \phi_{\max }=1+$ $2 \pi f_{\text {rf }} \alpha_{0}^{2} /\left(4 H_{0} \alpha_{2}\right)$. The result is real, if $\left|H_{0}\right|>$ $2 \pi f_{\mathrm{rf}} \alpha_{0}^{2} /\left(8 \alpha_{2}\right)$. At smaller values of $H_{0}$ bucket A merges with $\mathrm{B}$ and only buckets $\mathrm{B}$ and $\mathrm{C}$ remain, see Fig. 8 (bottom). The $\delta_{\max }$ term is calculated by solving the equation $H\left(\phi_{F}, \delta_{\max }\right)=H_{\mathrm{B}}$, where $\phi_{F}=0$ is the phase value of the stable FP at vanishing potential oscillation energy. The result is $\delta_{\max , B}^{2}=-2 \alpha_{0} / \alpha_{2}$. This maximum is only a function of $\alpha_{0}$ and $\alpha_{2}$, independent of the rf voltage. A similar analysis is done for buckets $\mathrm{A}$ and $\mathrm{C}$. The results are summarized in Table III.

In case of further reduced $\alpha_{2}$ values, the FP-part $\delta_{F}=\sqrt{-\alpha_{0} / \alpha_{2}}$ moves to larger values and buckets $\mathrm{B}$

TABLE III. Extreme values $\phi_{\max }, \delta_{\max }$ on the separatrix with $\alpha_{0}<0$ and $\alpha_{2}>0$. The value of the Hamiltonian on the separatrix is calculated at the given, unstable fixed points.

\begin{tabular}{lcc}
\hline \hline Bucket & $\operatorname{FP}\left(\phi_{F}, \delta_{F}\right)$ & $\cos \phi_{\max }$ \\
\hline A & $\left(\pi, \sqrt{-\alpha_{0} / \alpha_{2}}\right)$ & -1 \\
B & $(0,0)$ & $1+2 \pi f_{\mathrm{rf}} \alpha_{0}^{2} /\left(4 H_{0} \alpha_{2}\right)$ \\
C & $\left(\pi, \sqrt{-\alpha_{0} / \alpha_{2}}\right)$ & $-1-2 \pi f_{\mathrm{rf}} \alpha_{0}^{2} /\left(4 H_{0} \alpha_{2}\right)$ \\
\hline Bucket & $\operatorname{FP}\left(\phi_{F}, \delta_{F}\right)$ & $\delta_{\max }^{2}$ \\
\hline A & $\left(\pi, \sqrt{-\alpha_{0} / \alpha_{2}}\right)$ & $-\alpha_{0} / \alpha_{2}+\sqrt{-8 H_{0} /\left(2 \pi f_{\mathrm{rf}} \alpha_{2}\right)}$ \\
B & $(0,0)$ & $-2 \alpha_{0} / \alpha_{2}$ \\
C & $\left(\pi, \sqrt{-\alpha_{0} / \alpha_{2}}\right)$ & $-\alpha_{0} / \alpha_{2}$ \\
\hline \hline
\end{tabular}

and $\mathrm{C}$ become separated by a layer of unbounded phase motion. This situation is not further discussed here.

Following the definition of [34], a bucket size defined by the rf voltage is called an "rf bucket," if the size is defined by the $\alpha$ values it is called an " $\alpha$ bucket." The size of the buckets, given by the limits of momentum and phase acceptance presented in Table III, depend on a more complicated way on $\alpha$ values and rf voltage.

\section{COHERENT SYNCHROTRON RADIATION}

The goal of the short bunches produced by the low- $\alpha$ optics is the production of $\mathrm{THz}$ radiation. These short electron bunches emit coherent synchrotron radiation. Signals of the radiation are measured and analyzed in time and frequency domain at a dedicated $\mathrm{THz}$ beam line [44] of $64 \mathrm{mrad}$ (horizontal) x $43 \mathrm{mrad}$ (vertical) acceptance. In Fig. 11, upper and lower part, two types of $\mathrm{THz}$ signals in the time domain are shown, recorded by an InSb detector of $1 \mathrm{MHz}$ bandwidth. The rms bunch length was about 3 ps at $630 \mathrm{MeV}$ energy, an applied rf voltage of $250 \mathrm{kV}$ and $\alpha_{0}=4.6 \times 10^{-4}$. Bursting emission was achieved at $35 \mathrm{~mA}$ multibunch current. This emission type shows a strong time dependency. The emission can fluctuate periodically in the $\mathrm{kHz}$ range or stochastically. The second emission type, measured at $20 \mathrm{~mA}$, produces stable signals. These signals are constant in time. In the upper part, examples of periodic bursting $\mathrm{THz}$ signals are shown and in the lower part stable THz signals. On the left part, the signals are additionally mechanically chopped. The bursting signals can be seen in the chopped and unchopped mode, Figs. 11(a) and 11(b). The stable, steady state signals are of constant intensity within the time resolution of the InSb detector. The detector is ac coupled and the signals for stable $\mathrm{THz}$ are only visible, if they are chopped, Fig. 11(c). In the unchopped case, Fig. 11(d), the detector signal output is zero. Bursting THz signals are
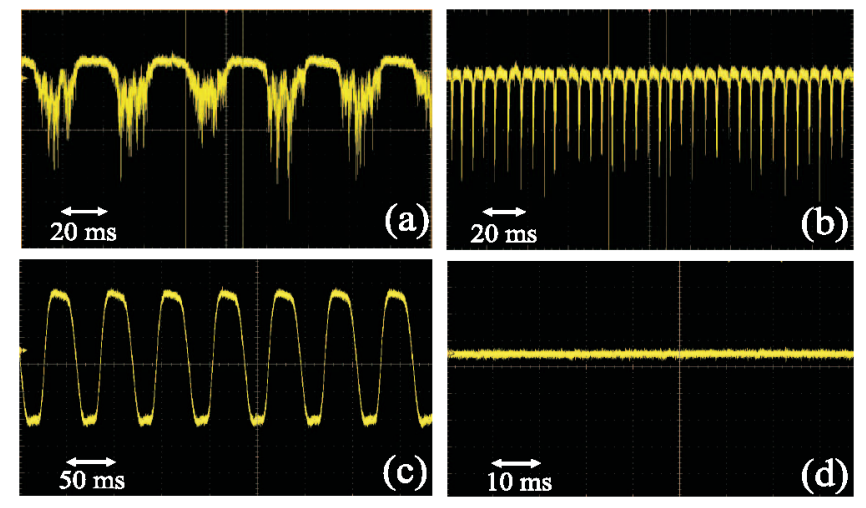

FIG. 11. Typical oscilloscope traces of bursting (top) and stable (bottom) THz signals. Parts (a) and (c) show mechanically chopped signals. Part (d) shows traces from stable THz signals, no intensity modulation within the $\mu \mathrm{s}$ time resolution of the detector is visible. 


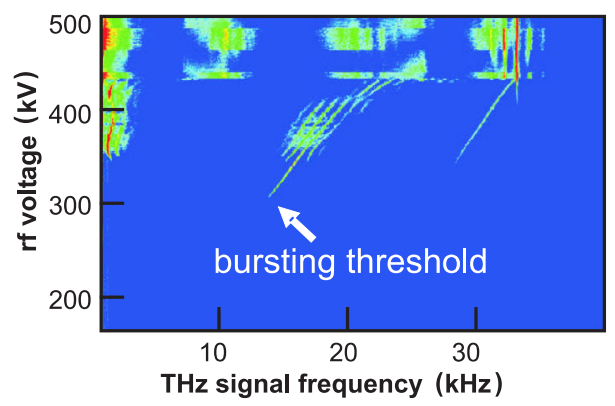

FIG. 12. Typical example of a "waterfall" plot of THz time signals. Shown are the temporal modulation of the $\mathrm{THz}$ signals as a function of the rf voltage amplitude. The bursting threshold is clearly visible.

produced above the threshold current of the bursting instability $[45,46]$. Steady state $\mathrm{THz}$ is of less power and emitted at bunch currents below this threshold.

This threshold is also dependent on the rf voltage. Another way to vary between stable or bursting $\mathrm{THz}$ is a change of the rf voltage amplitude at constant bunch current. This voltage change can be scanned over the range from stable to bursting $\mathrm{THz}$ emission. Figure 12 shows a typical example, measured at the MLS in single bunch mode of about $0.5 \mathrm{~mA}$ current, see also [47]. Clearly visible is the bursting threshold of the $\mathrm{THz}$ radiation. The bursting starts at $300 \mathrm{kV}$ with a sharp temporal oscillation frequency of $15 \mathrm{kHz}$. The signals of stable emission are not detected; for this a chopper is required. In any case, the $\mathrm{THz}$ radiation is modulated with the $6.25 \mathrm{MHz}$ bunch revolution rate, too fast to be resolved by the InSb detector. Some more recent results on bursting signals measured at the MLS are published in [48], as a first part of a detailed study.

In Fig. 13 the detected average $\mathrm{THz}$ power at the experimental port of the $\mathrm{THz}$ beam line is shown, as a function of the bunch current at a calculated zero current bunch length of $1 \mathrm{ps}(\mathrm{rms})$. The beam energy is $630 \mathrm{MeV}$ at an applied rf voltage of $450 \mathrm{kV}$. A maximum average value of about $60 \mathrm{~mW}$ is measured with a Thomas Keating power meter [49]. For currents above $15 \mathrm{~mA}$, the THz power does not scale with the square of the bunch current, expected only for bunches of constant shape. This indicates current dependent bunch lengthening effects.

The spectral content of the radiation was analyzed with a Vertex 80v Fourier transform spectrometer $[44,50]$. First results are presented in Fig. 14, measured with a room temperature deuterated triglycine sulfate detector and $6 \mu \mathrm{m}$ beam splitter. It was found that the spectrum ranges from $1.4 \mathrm{~cm}^{-1}$ to about $50 \mathrm{~cm}^{-1}$. The long wavelength limit (not shown in Fig. 14) is in agreement with estimations from standard cutoff formulas for the vacuum chamber [44,51]. The cutoff wavelength $\lambda_{\text {cutoff }}$ depends on the dipole bending radius $\rho$ and on the full chamber height $h$, $\lambda_{\text {cutoff }}=2 h \sqrt{h / \rho}$, where the small bending radius of the

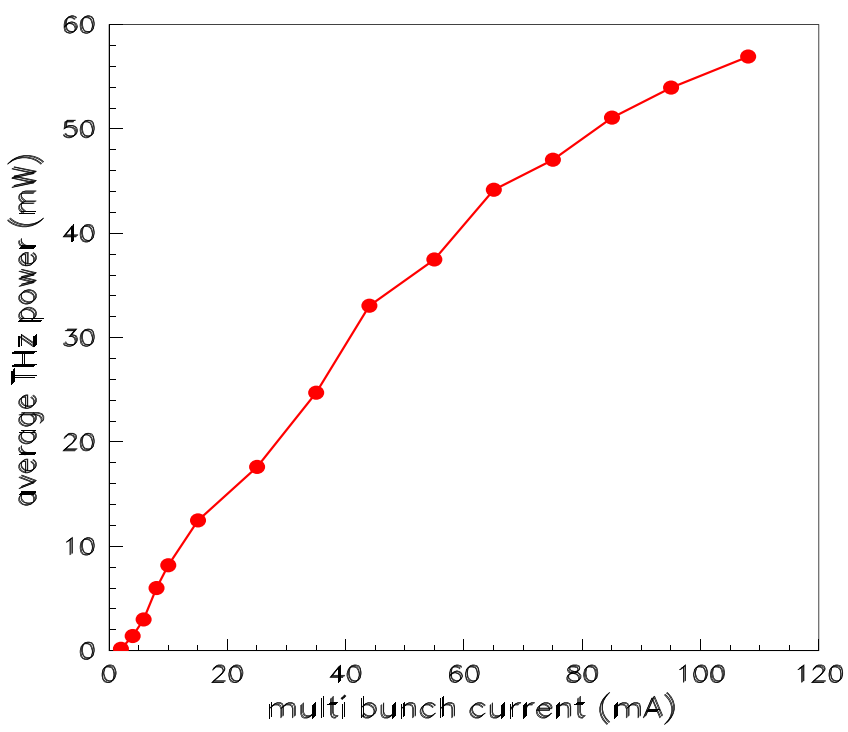

FIG. 13. Measured averaged $\mathrm{THz}$ power as a function of the multibunch current. A maximum of $60 \mathrm{~mW}$ power is reached.

MLS is advantageous to achieve the long wavelength range. The high frequency part is qualitatively similar to results achieved at BESSY II [8], in agreement with theoretical results discussed in $[3,9]$. The BESSY results are dependent on the machine parameters. The MLS results do fit within this range of variations. The spectrum and the emitted $\mathrm{THz}$ power are sensitive to trapped ions, as discussed in [52]. Similar effects are not observed at BESSY II.

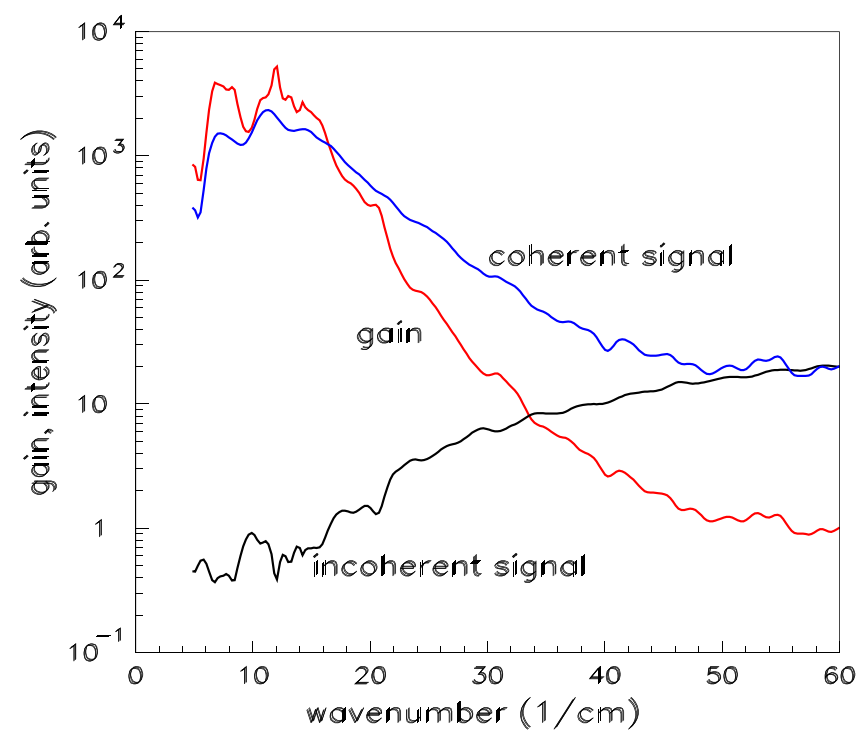

FIG. 14. Fourier transform $\mathrm{THz}$ spectrum, taken at $630 \mathrm{MeV}$, $100 \mathrm{~mA}$ beam current and $1 \mathrm{~cm}^{-1}$ resolution. In this example the power spectrum reaches beyond $50 \mathrm{~cm}^{-1}$ with a peak gain $>1000$. Based on the longitudinal bucket parameters, the calculated zero current bunch length is 1 ps. 


\section{SUMMARY}

The MLS is the first storage ring optimized for $\mathrm{THz}$ production. The low- $\alpha$ optics is very successfully tunable with the combination of three sextupole families and one octupole family. The minimum value of $|\alpha|$ is detected by the appearance of the double beam. Stable and bursting coherent $\mathrm{THz}$ is produced and the spectrum measured. Intense $\mathrm{THz}$ radiation of up to $60 \mathrm{~mW}$ is achieved. The spectrum reaches beyond $50 \mathrm{~cm}^{-1}$.

\section{ACKNOWLEDGMENTS}

This work was excellently supported by our HZB and PTB colleagues, in particular M. Abo-Bakr, K. BürkmannGehrlein, K. Holldack, P. Kuske, J. Rahn, and U. Schade. For fruitful discussions and active support we acknowledge the help by A. D. Bawagan (Carleton University, Canada), J. Corbett (SLAC, USA), H.-W. Hübers (DLR, Germany), I. P. S. Martin (Diamond, UK), and A.-S. Müller (ANKA, Germany).

[1] G. Wüstefeld, in Proceedings of the 11th European Particle Accelerator Conference, Genoa, 2008 (EPS-AG, Genoa, Italy, 2008), p. 26.

[2] Coherent Synchrotron Radiation in Storage Rings, ICFA Beam Dynamics Newsletter No. 35, edited by C. Biscari (2004), http://icfa-usa.jlab.org/archive/newsletter/icfa_bd_ nl_35.pdf.

[3] Micro Bunches Workshop, AIP Conf. Proc. No. 367, edited by E. B. Blum et al. (AIP, Upton, NY, 1995), Vol. 367.

[4] DOE-NSF-NIH, Opportunities in $\mathrm{THz}$ Science www .er.doe.gov/bes/reports/abstracts.html\#THz.

[5] G. P. Williams, Rep. Prog. Phys. 69, 301 (2006).

[6] M. Abo-Bakr, J. Feikes, K. Holldack, G. Wüstefeld, and H.-W. Hübers, Phys. Rev. Lett. 88, 254801 (2002).

[7] G. L. Carr et al., Nature (London) 420, 153 (2002).

[8] M. Abo-Bakr et al., Phys. Rev. Lett. 90, 094801 (2003).

[9] F. Sannibale et al., Phys. Rev. Lett. 93, 094801 (2004).

[10] R. Klein et al., Phys. Rev. ST Accel. Beams 11, 110701 (2008).

[11] B. Beckhoff et al., Phys. Status Solidi B 246, 1415 (2009).

[12] U. Schade et al., Synchrotron Radiat. News 20, 20 (2007).

[13] A. Schnegg et al., Phys. Chem. Chem. Phys. 11, 6820 (2009).

[14] J. B. Murphy and S. Krinsky, Nucl. Instrum. Methods Phys. Res., Sect. A 346, 571 (1994).

[15] D. Krämer et al., MLS Technical Design Report, BESSY GmbH, Berlin, Germany, 2003.

[16] J.M. Byrd et al., in Proceedings of the 9th European Particle Accelerator Conference, Lucerne, 2004 (EPSAG, Lucerne, 2004), p. 2436.

[17] F. Sannibale et al., J. Synchrotron Radiat. 15, 655 (2008).

[18] E.-S. Kim, Jpn. J. Appl. Phys. 46, 7952 (2007).
[19] C. Pellegrini et al., Nucl. Instrum. Methods Phys. Res., Sect. A 301, 27 (1991).

[20] A. Ando et al., J. Synchrotron Radiat. 5, 342 (1998).

[21] L. H. Thomas, Phys. Rev. 54, 580 (1938).

[22] H.F. Schopper, Advances of Accelerator Physics and Technologies (World Scientific, Singapore, 1993), p. 528.

[23] "Isochronous Apparatus, " picture is courtesy of the Museum of the History of Physics, University of Padua (Italy), www.unipd.it/musei/en/physic/collections.html, see also Museum of the History of Science, Florence (Italy).

[24] E. Weihreter, in Proceedings of the 11th European Particle Accelerator Conference, Genoa, 2008 (Ref. [1]), p. 2939.

[25] W. Anders, in Proceedings of the EPAC 1992 (Berlin, Germany, 1992), p. 798.

[26] H. Wiedemann, Particle Accelerator Physics II (SpringerVerlag, Berlin, 1995), Chap. 6.3.

[27] E. Forest, Beam Dynamics (Harwood Academic Publisher, Amsterdam, The Netherlands, 1998), Chap. 9.

[28] Y. Shoji, Phys. Rev. ST Accel. Beams 8, 094001 (2005).

[29] Y. Shoji, Phys. Rev. ST Accel. Beams 7, 090703 (2004).

[30] A. Ando and K. Takayama, in Proceedings of the PAC 1983, Santa Fe, 1983 [IEEE Trans. Nucl. Sci. 30, 2604 (1983)].

[31] K.-Y. Ng, Nucl. Instrum. Methods Phys. Res., Sect. A 404, 199 (1998).

[32] C. J. A. Corsten, Ph.D. thesis, Eindhoven University of Technology (Netherlands), 1982.

[33] H. Hama et al., Nucl. Instrum. Methods Phys. Res., Sect. A 329, 29 (1993).

[34] D. Robin, E. Forest, C. Pellegrini, and A. Amiry, Phys. Rev. E 48, 2149 (1993).

[35] H. Grote and F. Ch. Iselin, The MAD Program, Version 8.21 (CERN, Geneva, Switzerland, 1997).

[36] H. Nishimura et al., in Proceedings of the 9th European Particle Accelerator Conference, Lucerne, 2004 (Ref. [16]), p. 2182.

[37] Y. Shoji, in Proceedings of the 1st International Particle Accelerator Conference (IPAC'10 OC/ACFA, Kyoto, Japan, 2010), p. 289.

[38] J. Feikes et al., in Proceedings of the 9th European Particle Accelerator Conference, Lucerne, 2004 (Ref. [16]), p. 2290 .

[39] J. Feikes et al., in Proceedings of the 10th European Particle Accelerator Conference, Edinburgh, Scotland, 2006 (EPS-AG, Edinburgh, 2006), p. 3308.

[40] J. B. Murphy and S. L. Kramer, Phys. Rev. Lett. 84, 5516 (2000).

[41] I. P. S. Martin et al., in Proceedings of the 23rd Particle Accelerator Conference, Vancouver, Canada, 2009 (IEEE, Piscataway, NJ, 2009), p. 3769.

[42] www.scholarpedia.org/article/Duffing_oscillator.

[43] C. Koschitzki et al., in Proceedings of the 1st International Particle Accelerator Conference (Ref. [37]), p. 894.

[44] R. Müller et al., in Proceedings of the 23rd Particle Accelerator Conference, Vancouver, Canada, 2009 (Ref. [41]), p. 2288.

[45] G. Stupakov and S. Heifets, Phys. Rev. ST Accel. Beams 5, 054402 (2002). 
[46] M. Venturini and R. Warnock, Phys. Rev. Lett. 89, 224802 (2002).

[47] P. Kuske, in Proceedings of the 23rd Particle Accelerator Conference, Vancouver, Canada, 2009 (Ref. [41]), p. 4682.

[48] G. Wüstefeld et al., in Proceedings of the 1st International Particle Accelerator Conference (Ref. [37]), p. 2508.
[49] www.terahertz.co.uk.

[50] www.brukeroptics.com.

[51] R. L. Warnock, Report No. SLAC-PUB-5375, 1990.

[52] J. Feikes et al., in Proceedings of the 23rd Particle Accelerator Conference, Vancouver, Canada, 2009 (Ref. [41]), p. 1090. 